\title{
Therapy Monitoring and Patient Evaluation with Social Robots
}

\author{
Alejandro Martín \\ Universidad Carlos III de \\ Madrid \\ alejandro.martin@uam.es \\ Ángel García-Olaya \\ Universidad Carlos III de \\ Madrid \\ agolaya@inf.uc3m.es
}

\author{
José C. González \\ Universidad Carlos III de \\ Madrid \\ josgonza@inf.uc3m.es \\ Fernando Fernández \\ Universidad Carlos III de \\ Madrid \\ ffernand@inf.uc3m.es
}

\author{
José C. Pulido \\ Universidad Carlos III de \\ Madrid \\ jcpulido@inf.uc3m.es \\ Cristina Suárez \\ Hospital Virgen del Rocío, \\ Sevilla \\ cristina.suarez.exts@junta \\ deandalucia.es
}

\begin{abstract}
Social robots have a great potential. With high movement capabilities and large computational capacity, they allow to perform varied tasks that were usually conducted by humans. One of these tasks are physical therapies, where a therapist guides a patient through the realisation of a set of exercises. A robot, equipped with a sophisticated artificial vision system, can conduct these therapies and evaluate the patient movements. In this paper, we present a system that allows the therapist to design a complete therapy to be carried out by the robot, to start each session with the robot, to evaluate the patient condition over the therapy and to generate reports at the end of a session.
\end{abstract}

\section{Categories and Subject Descriptors}

I.2.9 [Artificial Intelligence]: Robotics

\section{General Terms}

Design, Human Factors, Measurement

\section{Keywords}

Social Robot, QUEST, Therapy, Cerebral Palsy

\section{INTRODUCTION}

Usually, therapies for the treatment of patients with cerebral palsy involve a therapist who guides the patient through the realisation of a series of training exercises. At the same time, the therapist assess the range of movement of the joints affected evaluating the maximum angle achieved.

This is the most common approach in the hospital environment. Due to the young age of children that have to cope with rehabilitation sessions, which can start in the first two years of life, several problems arise: lack of attention, boredom or feelings of hopelessness become apparent in the course of the therapy. Today, the development of robots with human aspect and high movement abilities has encouraged its use in this type of tasks. Social robots have proven to be an effective means to capture patient attention and build a more pleasant environment, where the social capabilities they provide allow a strong interaction [7]. This is the idea and objectives of NAOTherapist, a system where a NAO robot and a Microsoft Kinect camera are used to lead the therapy and monitor the patient, respectively. Fig. 1 shows a NAOTherapist session. A computerised system, the camera and the robot are connected to, controls everything regarding the execution of a session, but also, by means of a graphical application and using Automated Planning, allows the therapist to design the therapy, based on several clinical parameters. NAOTherapist is part of the Therapist project [3].

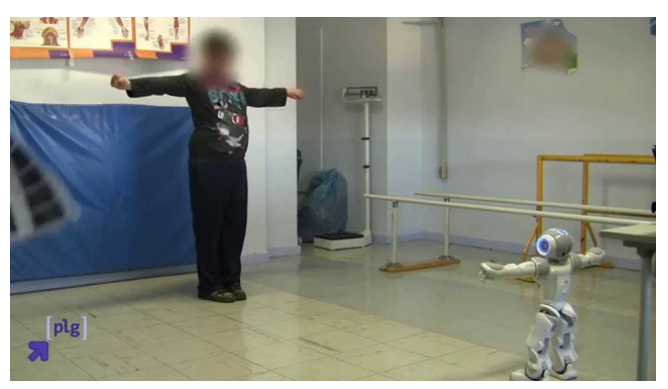

Figure 1: NAOTherapist session

Comparing a traditional rehabilitation session to another one where social robots are used, there is a gap not clearly solved. It deals with patient evaluation, not only for the purpose of deciding if a patient position is correct according to the artificial vision system data, but also for establishing the patient's medical condition. This is a really important part of therapies or any other medical test.

In rehabilitation therapies for patients with cerebral palsy, evaluation methods such as QUEST [5] (Quality of Upper Extremity Skills Test) or Melbourne Assessment are used. Generally, these metrics measure the range of motion of the 
patient's joints. A report based on the metric is completed by the therapist after the session. In the case of QUEST, it is composed of seven domains. The first four are involved in the calculation of the scale score. Each domain has a list of items defining postures that the patient has to adopt.

Having an artificial vision system, which provides detailed information about the patient position, it is possible for the system to automatically fill the clinical report associated with one of these metrics. This is one of the purposes of this work, to develop a system that automatically completes the report of a standardised metric and also allows the therapist to manually change the values using a graphical application. We have selected QUEST to asses patients in the therapies because it is specifically designed for evaluation of upper limbs of patients with cerebral palsy and it can be applied to small children, which are the target population of NAOTherapist.

This paper is organized as follows. In Section 2, we analyse related work, in Section 3 we define our solution, explaining each part that composes it, such as the session execution, session monitoring, patient evaluation and reports generation modules and Section 4 presents some conclusions.

\section{RELATED WORK}

In addition to the NAOTherapist project, there is more literature in the same direction. However, automatic patient evaluation using clinical metric is not their main objective. For example, a similar approach to NAOTherapist is taken in [8], where NAO robots and a Microsoft Kinect camera are the elements in common. The authors tackle the patient evaluation problem recording videos that afterwards are revised by the therapists, which are required to fill manually a simple metric designed by the authors. Humanoid robots are also employed in [11], but no patient evaluation method based on a clinical metric is followed.

Non-humanoid robotic platforms have been also used for this type of rehabilitation. Patient evaluation with a standard assessment procedure such as Fugl-Meyer[6] is compatible with this type of robots [4]. The main difference between this and our approach is the use of a robotic platform which needs physical contact (data are obtained from the movement of a manipulator), unlike in the architecture of NAOTherapist where there is no physical contact.

In other researches, authors use a webcam to analyse the patient position [2]. Due to the nature of the data collected from this camera, which are images without information about the patient position, the research focuses on the processing of the data.

The use of clinical metrics directly implemented into a robotic architecture is a new area of research where there has been limited previous work. Data extracted from a joystick handled by the patient are used in [1], where the QUEST report is filled manually by the physician. In contrast, we focus on the implementation of an autonomous system where the QUEST report is, at least partially, automatically filled.

Concerning the scales to evaluate patients, there are several ones that can be applied. The choice relies on multiple factors which have to be considered. Each metric can be focused in a specific part of the body or it can be associated with a particular disease. [9] describes these scales and classifies them into three categories: condition-specific tests, generic tests and questionnaires. The first is the closest section to NAOTherapist objectives, because these metrics work with a specific section of the body, the upper limbs. QUEST[5] and Melbourne Assessment are part of this category. Generic tests encompass wider evaluations, where the illness affects several parts of the body whereas questionnaires are simpler and can be filled by the parents.

\section{SYSTEM DESIGN}

A session of NAOTherapist involves multiple components that have to be executed together. In Fig. 2 the execution cycle of a session is shown. The artificial vision system, the robot, the planner of all the exercises that the patient has to perform or the automatic patient evaluation system, take part of a session. Given the complex nature of the system components and the many interactions among them, it is of paramount importance to give the therapist an easy to use interface covering all the process, from the planning of the therapy to the reports elaboration, including monitoring of session execution.

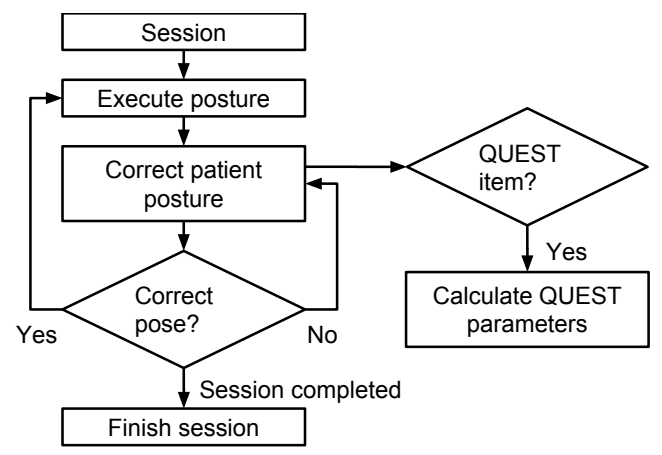

Figure 2: NAOTherapist therapy execution cycle

We have developed a system that can be operated by the therapist in order to manage patient's data, sessions and reports, to design a plan of sessions and to execute each session. For this purpose we have created an interface with multiple tabs, that match each phase of a session. The first tab allows the therapist to manage patients and to generate reports once a session has finished. The Therapies tab allows the therapist to automatically design a whole therapy for a given patient. A complete therapy is composed of a number of sessions consisting of several exercises, each one requiring the patient to take a set of poses. Different parameters to adapt the therapy to each patient can be configured. The process of scheduling a list of sessions for a patient, including a set of exercises for each of them, was previously described [10] and is out of the scope of this paper.

Once the therapy is planned, the therapist can start its execution. At this point, different processes start, connecting the Kinect sensor and the NAO robot with a central component module, which handles them to perform a complete session of NAOTherapist. In the next subsections we describe each of these steps. 


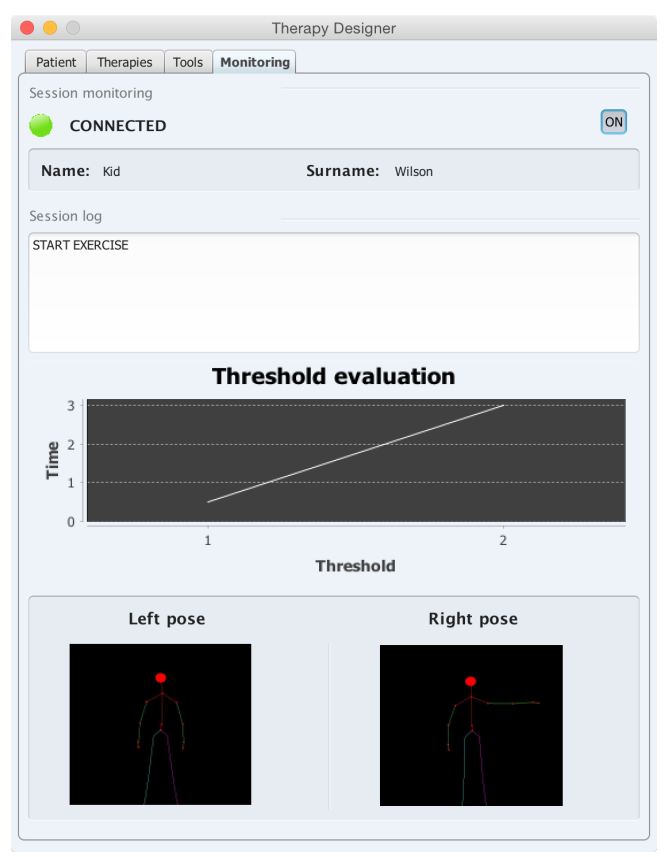

Figure 3: NAOTherapist monitoring

\subsection{Session monitoring}

A session undertaken with the NAO robot does not require the presence of the therapist. The robot leads the whole therapy without the participation of the therapist, asking the child to take a given pose, monitoring it and encouraging him/her when difficulties arise. If the therapist decides to absent from the therapy room, a system to monitor what it is happening during the session is needed. We have designed and implemented a subsystem in our application for this purpose (Fig. 3). This tab, receiving information from the central component of the NAOTherapist architecture, displays the patient's name, a console with all the actions that the robot performs, a graphic with the evolution of a parameter that indicates how well the patient is doing the exercise (how close his/her joints' angles are to target ones) and the poses the patient has to do.

\subsection{Poses evaluation}

Each pose that the patient has to adopt is captured by the artificial vision system. The generated data, composed by a set of angles of the upper limbs, are used to decide whether the pose is correct or not. Thus far, this process has used a mathematical calculation to take the decision; a distance between the angles shown by the robot and the real angles that the patient adopts. This process, however, does not take into account the therapist's expert knowledge, who is able to consider other relevant information to take the decision. In order to solve this problem, we have used the J48 machine learning algorithm, which after a training process, generates a decision tree. This type of model has an understandable representation, which is a essential feature in a medical domain. We have trained this model separately for each limb using the decisions generated by the mathematical model to evaluate whether machine learning can be used to mimic it. The minimum number of instances per leaf significantly affects the quality of the model. Fig. 4 shows the hit rate, the number of leafs and the number of nodes for each value taken for this parameter. Best value is 20 , due to the sharp decline of the number of nodes and leafs (that simplifies the model) while maintaining a high hit rate, measured as the proportion of poses correctly classified as correct or incorrect.

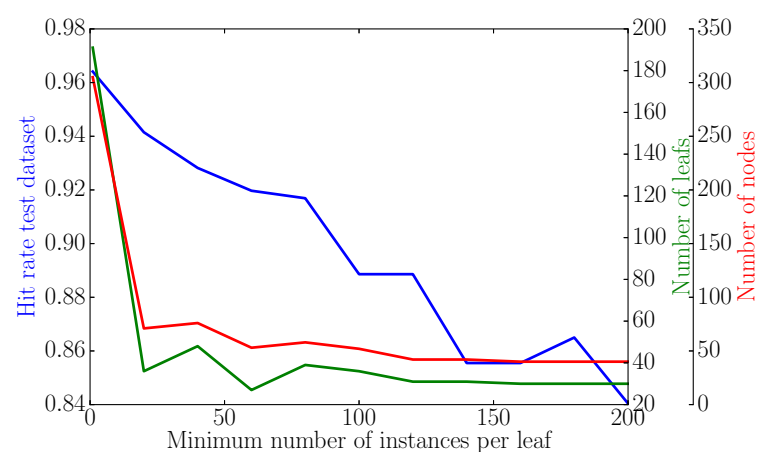

Figure 4: Results of experiments performed using J48 for the right limb

\subsection{Patient evaluation}

Filling the report of the QUEST scale is a task the therapist uses to do manually, measuring the range of movement in the joints of the patient. However, the vision component of NAOTherapist is able to measure every upper limbs angle, allowing to fill the report automatically. One example of these angles is shown in Figure 5. Both items are evaluated by measuring the range of motion, deciding whether the patient is able to move the limb up to the middle of the whole angle, $90^{\circ}$ or up the complete angle.

This process can be easily performed with the information about angles retrieved from the Kinect sensor. However, only a limited number of items of the scale can be calculated due to the robot and camera limitations. For instance, any measure related to fingers movement is out of scope, because of the lack of detail of the Kinect camera and the impossibility of moving the robot fingers individually.
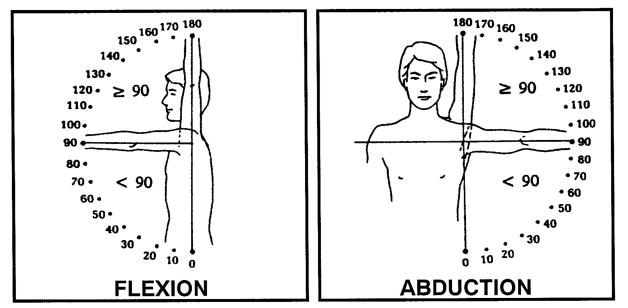

Figure 5: Example of items in QUEST

We have implemented a system able to calculate four items of the first domain of QUEST, called Dissociated Movements. Two items belong to the shoulder section while the other two belong to the elbow section. Despite the fact that the evaluated items are only a small part of the whole scale, our objective has been to develop an easily expandable, fully functional and easy to use system. If a more accurate camera or a different robot were used more report items could be automatically filled. 


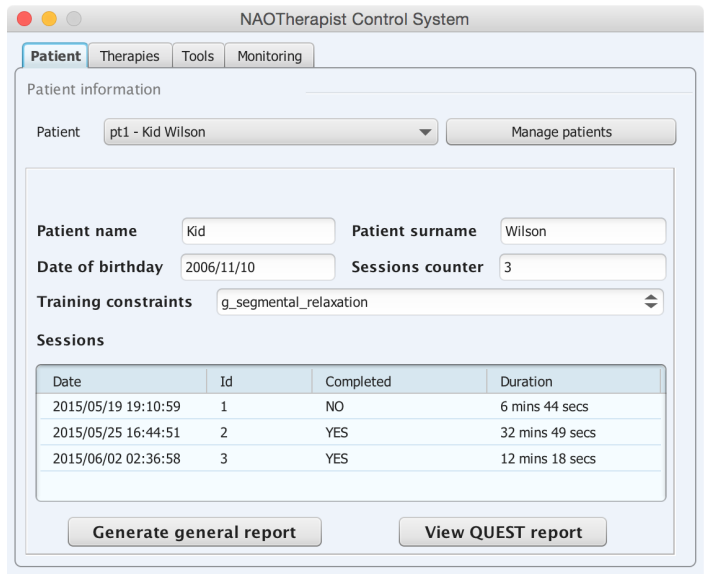

Figure 6: NAOTherapist sessions and reporting

The system includes a graphical interface allowing the therapist to view the automatically generated QUEST report, with the four mentioned above items filled. Using this tool, the therapist can change the value of any item, manually filling all the sections. The metric score is also automatically calculated by the computer. Once the therapist has completed the QUEST report, the application generates a PDF document, with similar aspect to the original report.

\subsection{Sessions management and reporting}

Once a session is finished the system stores all the data related to it. The therapist can access to a history of past sessions for each patient (Fig. 6). Selecting one patient, the therapist can create a report containing detailed information about a session. To internally build this file, during the session, an XML file is saved with all the postures taken by the patient. When the therapist asks for the report, the JasperReports Library ${ }^{1}$ is used to generate the document, reading the XML file to retrieve the necessary data. This general report shows all the postures adopted by the patient, detailing the principal angles of each limb: shoulder flexion, shoulder abduction, elbow flexion and elbow extension.

\section{CONCLUSIONS}

Physical therapies are considered a very important activity to treat diseases such as cerebral palsy. Helping the patients to extend the range of movement of their joints is a essential task to improve their condition. The wider the range of movement achieved, the higher the possibility to perform daily tasks during their lives. Social robots can be a useful tool to guide these therapies. However, the lack of extended scales for patient evaluation prevents them from being implemented in hospitals. Our solution NAOTherapist, a platform which integrates robots with a vision system, is able to automatically conduct rehabilitation sessions, evaluate the patient according to a widely used clinical scale and also allows the therapist to manage the created reports. Currently, the system is being tested in a real-world environment. The real data about poses classification performed by therapists will be used in future work to test the learning models.

\footnotetext{
${ }^{1}$ http://community.jaspersoft.com/
}

\section{ACKNOWLEDGMENTS}

This work is partially funded by grant TIN2012-38079-C0302 of Spanish Ministerio de Economía y Competitividad.

\section{REFERENCES}

[1] V. Aharonson and H. I. Krebs. Prediction of response to robot-aided motor neuro-rehabilitation of children with cerebral palsy. Biomedical Signal Processing and Control, 7(2):180-184, Mar. 2012.

[2] D. A. Brooks and A. M. Howard. Quantifying upper-arm rehabilitation metrics for children through interaction with a humanoid robot. Applied Bionics and Biomechanics, 9(2):157-172, 2012.

[3] L. V. Calderita, P. Bustos, C. S. Mejías, F. Fernández, and A. Bandera. Therapist: Towards an autonomous socially interactive robot for motor and neurorehabilitation therapies for children. In Proceedings of the 7th International Conference on Pervasive Computing Technologies for Healthcare, pages 374-377, 2013.

[4] R. Colombo, F. Pisano, S. Micera, A. Mazzone, C. Delconte, C. M. Carrozza, P. Dario, and G. Minuco. Robotic techniques for upper limb evaluation and rehabilitation of stroke patients. Neural Systems and Rehabilitation Engineering, IEEE Transactions on, 13(3):311-324, 2005.

[5] C. Dematteo and N. Pollock. Quality of Upper Extremity Skills Test. Physical Occupational Therapy in Pediatrics, 13:1-18, 1992.

[6] A. R. Fugl-Meyer, L. Jääskö, I. Leyman, S. Olsson, and S. Steglind. The post-stroke hemiplegic patient. 1. a method for evaluation of physical performance. Scandinavian journal of rehabilitation medicine, 7(1):13-31, 1974

[7] M. A. Goodrich and A. C. Schultz. Human-robot interaction: a survey. Foundations and trends in human-computer interaction, 1(3):203-275, 2007.

[8] A. Guneysu, R. Siyli, and A. Salah. Auto-evaluation of motion imitation in a child-robot imitation game for upper arm rehabilitation. Robot and Human Interactive Communication, 2014 RO-MAN: The 23rd IEEE International Symposium on, pages 199-204, 2014.

[9] K. Klingels, E. Jaspers, A. Van de Winckel, P. De Cock, G. Molenaers, and H. Feys. A systematic review of arm activity measures for children with hemiplegic cerebral palsy. Clinical rehabilitation, 24(10):887-900, Oct. 2010

[10] J. C. Pulido, J. C. González, A. González-Ferrer, J. García, F. Fernández, A. Bandera, P. Bustos, and C. Suárez. Goal-directed generation of exercise sets for upper-limb rehabilitation. In Proc. of the Workshop on Knowledge Engineering for Planning and Scheduling, 2014.

[11] S. Shamsuddin, H. Yussof, L. Ismail, F. A. Hanapiah, S. Mohamed, H. A. Piah, and N. I. Zahari. Initial response of autistic children in human-robot interaction therapy with humanoid robot NAO. In 2012 IEEE 8th International Colloquium on Signal Processing and its Applications, pages 188-193. IEEE, Mar. 2012. 